\title{
Quantum Simulations of Curved Space
}

\author{
A heptagonal-lattice superconducting circuit, and the mathematics that \\ describe it, provide tools for studying quantum mechanics in curved \\ space.
}

\section{By Rachel Berkowitz}

$\Lambda$ ccording to John Wheeler's summary of general relativity, "space-time tells matter how to move; matter tells space-time how to curve." How this relationship plays out at the quantum scale is not known, because extending quantum experiments to curved space poses a challenge. In 2019, Alicia Kollár and colleagues at Princeton University met that challenge with a photonic circuit that represents the negatively curved space of an expanding universe [1]. Now, Igor Boettcher and colleagues at the University of Maryland, College Park, describe those experiments with a new theoretical framework [2]. Together, the studies offer a toolkit for studying quantum mechanics in curved space that could help answer fundamental questions about cosmology.

In a universe that expands at an accelerating rate, space curves away from itself at every point, producing a saddle-like, hyperbolic geometry. To project hyperbolic space onto a plane, Kollár's team etched a centimeter-sized chip with
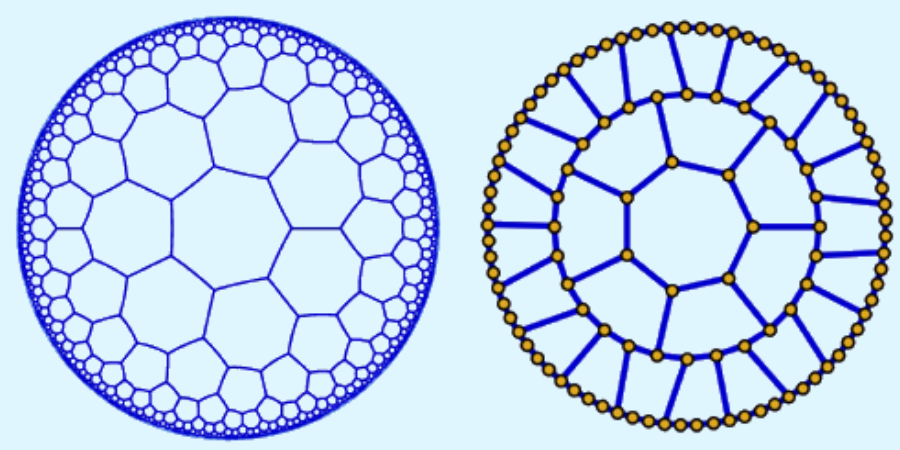

Credit: I. Boettcher et al. [2] superconducting resonators arranged in a lattice of heptagonal tiles. By decreasing the tile size toward the edge of the chip, the researchers reproduced a perplexing property of hyperbolic space: most of its points exist on its boundary. As a result, photons moving through the circuit behave like particles moving in negatively curved space.

Using tools from graph theory and differential geometry, Boettcher and colleagues now use the Princeton team's hyperbolic lattice to shape the continuous space underlying a quantum field theory. Unlike in earlier models, curvature in this field theory arises naturally from the lattice geometry rather than being imposed by a wave equation. The researchers use their framework to describe photon dynamics within the photonic circuit, and they compute key observables that are borne out by the experiment.

Boettcher says that the work could help answer questions about quantum gravity, quantum information, and black hole physics. Applying concepts from geometry to graph theory problems could also reveal complex relationships in communication networks or machine-learning applications.

Rachel Berkowitz is a Corresponding Editor for Physics based in Seattle, Washington, and Vancouver, Canada.

\section{REFERENCES}

1. A. J. Kollár et al., "Hyperbolic lattices in circuit quantum electrodynamics," Nature 571, 45 (2019).

2. I. Boettcher et al., "Quantum simulation of hyperbolic space with circuit quantum electrodynamics: From graphs to geometry," Phys. Rev. A 102, 032208 (2020). 